University of New Hampshire

University of New Hampshire Scholars' Repository

\title{
Diffuse Galactic continuum emission: Recent studies using COMPTEL data
}

\author{
A W. Strong \\ Max-Planck-Institut für extraterrestriche Physik \\ R Diehl \\ Max-Planck-Institut für extraterrestriche \\ V Schonfelder \\ Max-Planck-Institut für extraterrestrische Physik \\ K Bennett \\ ESTEC \\ Mark L. McConnell \\ University of New Hampshire - Main Campus, mark.mcconnell@unh.edu
}

See next page for additional authors

Follow this and additional works at: https://scholars.unh.edu/ssc

Part of the Astrophysics and Astronomy Commons

\section{Recommended Citation}

Diffuse Galactic continuum emission: Recent studies using COMPTEL data Strong, A. W. and Diehl, R. and Schönfelder, V. and Bennett, K. and McConnell, M. and Ryan, J., AIP Conference Proceedings, 410, 1198-1202 (1997), DOI:http://dx.doi.org/10.1063/1.53932

This Conference Proceeding is brought to you for free and open access by the Institute for the Study of Earth, Oceans, and Space (EOS) at University of New Hampshire Scholars' Repository. It has been accepted for inclusion in Space Science Center by an authorized administrator of University of New Hampshire Scholars' Repository. For more information, please contact Scholarly.Communication@unh.edu. 


\section{Authors}

A W. Strong, R Diehl, V Schonfelder, K Bennett, Mark L. McConnell, and James M. Ryan 


\section{AIP $\mid$ proceedings}

\section{Diffuse Galactic continuum emission: Recent studies using}

COMPTEL data

A. W. Strong, R. Diehl, V. Schönfelder, K. Bennett, M. McConnell, and J. Ryan

Citation: AIP Conference Proceedings 410, 1198 (1997); doi: 10.1063/1.53932

View online: http://dx.doi.org/10.1063/1.53932

View Table of Contents:

http://scitation.aip.org/content/aip/proceeding/aipcp/410?ver=pdfcov

Published by the AIP Publishing

\section{Articles you may be interested in}

Galactic diffuse $\mathrm{y}$-ray emission at $\mathrm{TeV}$ energies and the ultra-high energy cosmic rays

AIP Conf. Proc. 410, 1203 (1997); 10.1063/1.54102

Diffuse $50 \mathrm{keV}$ to $10 \mathrm{MeV}$ gamma-ray emission from the inner Galactic ridge AIP Conf. Proc. 410, 1193 (1997); 10.1063/1.54101

$26 \mathrm{Al}$ constraints from COMPTEL/OSSE/SMM data AIP Conf. Proc. 410, 1104 (1997); 10.1063/1.54089

COMPTEL spectral study of the inner galaxy

AIP Conf. Proc. 410, 1074 (1997); 10.1063/1.54085

Diffuse galactic continuum radiation

AIP Conf. Proc. 410, 192 (1997); 10.1063/1.54029 


\title{
Diffuse Galactic Continuum Emission: Recent Studies using COMPTEL Data
}

\author{
A. W. Strong*, R. Diehl*, V. Schönfelder*, \\ K. Bennett ${ }^{\ddagger}$, M. McConnell ${ }^{\ddagger}$, J. Ryan ${ }^{\ddagger}$ \\ * Max-Planck-Institut für extraterrestrische Physik, D-85740 Garching, Germany \\ ${ }^{\dagger}$ Astrophysics Division, ESTEC, 2200 AG Noordwijk, The Netherlands \\ ${ }^{+}$University of New Hampshire, Durham NH 03824, U.S.A.
}

\begin{abstract}
COMPTEL full sky maximum entropy maps using 5 years of data have been produced using background estimates based on high-latitude observations. The Galactic diffuse emission can be studied using latitude and longitude profiles from these maps. Direct comparison of profiles with theoretical models is illustrated for the $10-30 \mathrm{MeV}$ range. We demonstrate the presence of a broad latitude component, consistent with results from model-fitting studies. The method is also used to obtain model-independent broad-band spectra of the Galactic emission.
\end{abstract}

\section{INTRODUCTION}

The COMPTEL all-sky survey, containing over 5 years of mission data, has previously been used to generate whole-sky maps in the $1-30 \mathrm{MeV}$ range [1-3]. These images are generated using the maximum entropy method. Up to now these maps have mainly been used qualitatively to give a large-scale panorama of the gamma-ray sky at these energies. It is however desirable to try to make quantitative use of maximum entropy images since the intensity distribution can be compared directly with models and with images at other energies from other experiments. This provides an alternative to model fitting and gives a more direct visualization of the information coming from the data; and it is a model-independent technique. This approach has been pursued and validated for the case of $1.8 \mathrm{MeV}$ line emission $[4,5,7]$, where the background is derived from independent measurements at adjacent energies. The high background of COMPTEL makes imaging of extended continuum structure a challenging task. Previous all-sky continuum imaging studies [1-3] have derived the background model by a smoothing procedure applied to the same data; while this produces visually useful images, their quantitative use is limited due to the 
loss of signal from the smoothing process. Another method [8] is to make use of the observational data at high Galactic latitude, where little Galactic emission is expected, to derive estimates of the instrumental background structure. This method has the advantage of being largely independent of the data being analysed, hence reducing loss of signal. It is thus well adapted to studying extended structures. Time-dependence of the background at energies below $10 \mathrm{MeV}$ is difficult to incorporate however, and remains an uncertainty in this study. It is possible to handle these effects and this is foreseen in future work.

\section{METHOD}

COMPTEL data from CGRO Cycles 1-5 (199 observations) were used in the standard energy ranges $1-3,3-10$ and $10-30 \mathrm{MeV}$ (the 'ON' dataset). The data from 23 high-latitude observations $\left(|b|>50^{\circ}\right)$ were combined in the instrument coordinate system (the 'OFF' dataset); the background estimate in each of the $\mathrm{ON}$ datasets was obtained using a bicubic spline interpolation in the OFF dataset. These OFF counts were normalized to the ON dataset for each observation and then combined into one global background model for the entire survey. A corresponding global dataset was produced for the ON events and for the COMPTEL response.An additional factor in the range $0.90-0.95$ is applied to the background estimate to ensure that ON exceeds $\mathrm{OFF}$ everywhere in the dataspace; this procedure has only a second-order effect on the intensity distribution. The COMPTEL full sky maximum entropy software [1] was then used to produce the images. In order to evaluate the technique, simulations using model images of the Galaxy were performed. While these simulations are idealized they show that large-scale structure is recovered reliably, including the correct intensity scale, provided high iterations of the maximum-entropy solution are used. These high iterations approach the maximum-likelihood solution and hence are over-structured on small scales, and must be averaged over several degrees in $l$ or $b$ to obtain reliable profiles of large-scale structure. Note that these are the scales in which we are interested for diffuse emission studies. An important problem is the assignment of zero level, since this is by definition not determined in such a method. This degree of freedom is left when comparing with models or deriving spectra.

\section{RESULTS}

Fig 1 shows latitude and longitude profiles for the inner galaxy, averaged over $|b|<5^{\circ}$ for the longitude profile and $|l|<30^{\circ}$ for the latitude profile. The error bars are estimates from the observed fluctuations in the profiles at intermediate galactic latitudes where the celestial emission is weak. For comparison, the profile produced by a fit in the COMPTEL dataspace to a combination of narrow (gas) and broad component (IC) models is also shown, 

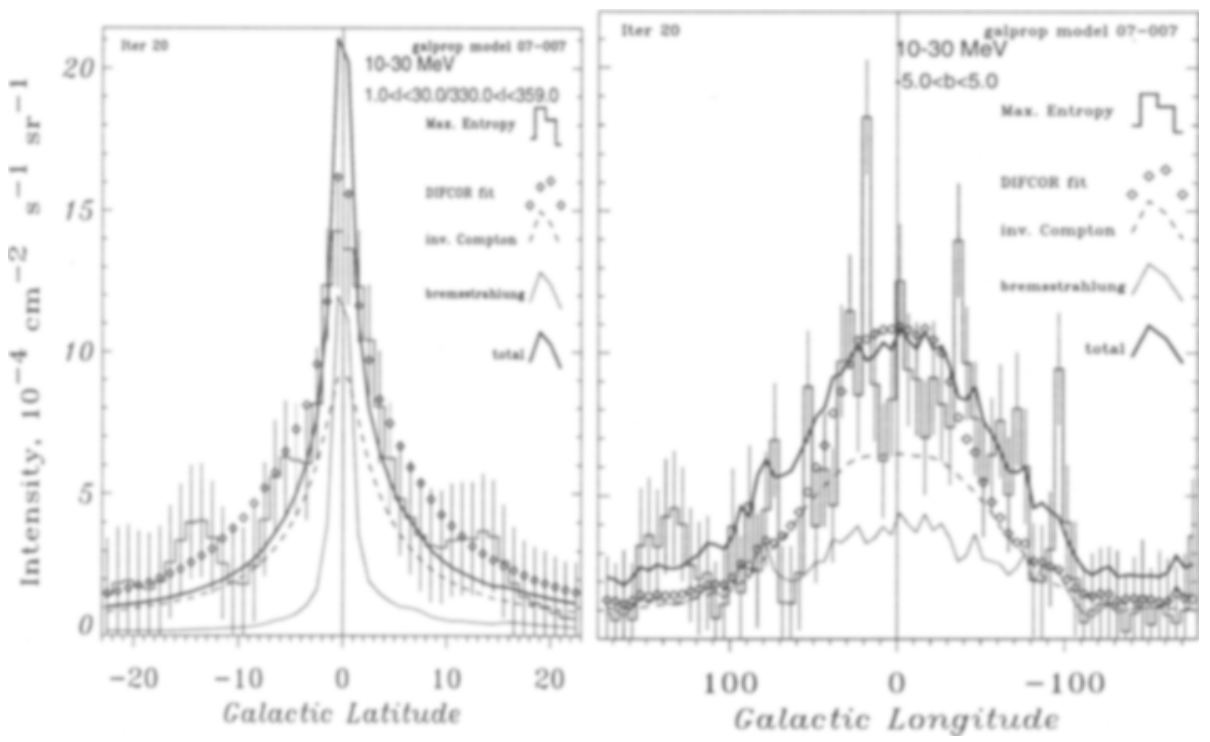

Fig 1. Latitude and longitude profiles of COMPTEL maximum entropy images are shown by the histograms. The intensity is averaged over $330^{\circ}<l<$ $30^{\circ}$ for the latitude profiles and $|b|<5^{\circ}$ for the longitude profiles. The profiles based on a two-component model fit to the data is also shown (diamonds). For comparison, emission predicted in [10] is shown, from bremsstrahlung (lower full line), inverse Compton (dashed line) and the total (full line).

the amplitudes of the two components being free parameters; this modeldependent profile agrees in overall shape with the maximum entropy profile. This is an important demonstration of the the reliability of the method, since it illustrates the degree of consistency between model-dependent and modelindependent approaches. We also compare with a detailed physical model for the emission $[9,10]$ in which a narrow bremsstrahlung and a broad inverse Compton component contribute roughly equally. This gives immediately an idea of how well the data supports such a model. The similarity in overall shape shows that such a model can reproduce the main features of both the latitude and longitude profiles. Clearly there is more structure present than in the model, due in part to unidentified point sources such as that at $l=18^{\circ}$ (other peaks are due to the Vela pulsar at $l=262^{\circ}$ and the Crab at $l=185^{\circ}$ ). Study of such distributions shows clearly in what respects the model fails to fit the data and suggests where improvements can be made. For example the particular model illustrated gives intensities which are rather too high suggesting that a slight decrease in the normalization of the cosmic-ray electron spectrum would fit better the lower envelope, with the localized excesses attributed to point sources. The difficulty in determining the absolute zero level is still a limiting factor which prevents a unique solution at present. 

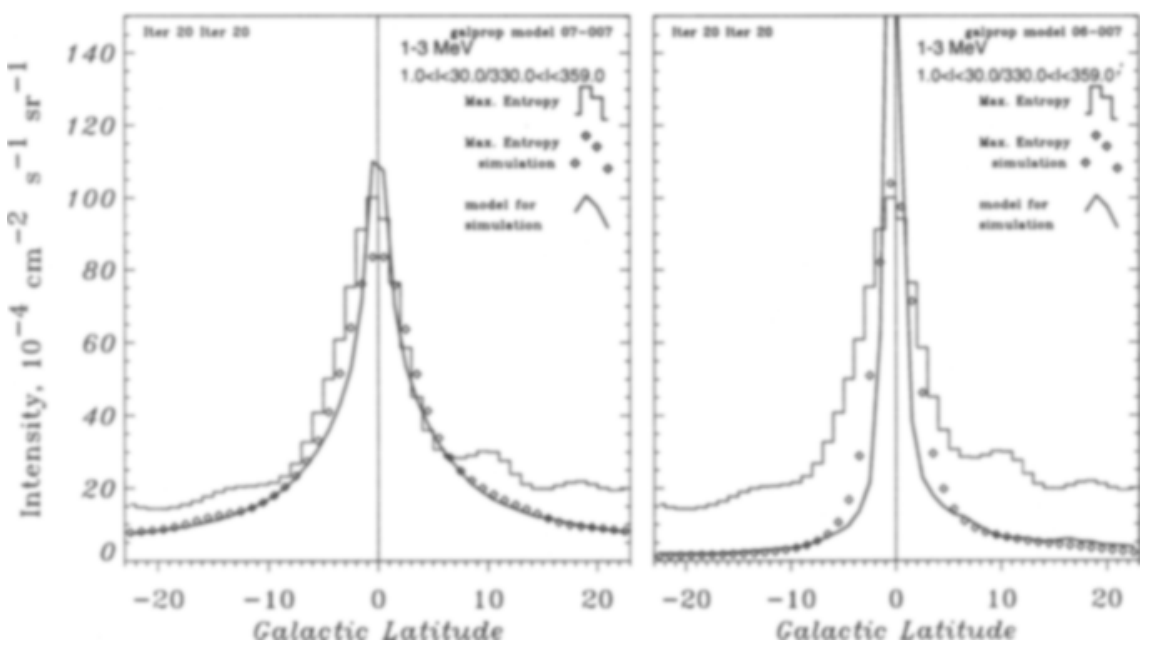

Fig. 2 Latitude profiles from maximum entropy images for $1-3 \mathrm{MeV}$, averaged over $330^{\circ}<l<30^{\circ}$. The histogram is from COMPTEL data, the diamonds are simulations based on (a) a broad (IC-like) latitude distribution (b) a narrow (gas-like) latitude distribution. The continuous curve is the model distribution used for the simulations.

For the energy range 1-3 MeV, we have made simulations for narrow and broad latitude distributions (Fig 2). These demonstrate that we expect to be able to distinguish between these on the basis of the images. The COMPTEL latitude profile resembles rather better the simulated result for the broad profile. Quantitatively the wide profile yields a factor 0.73 smaller unweighted minimum $\chi^{2}$ than the narrow one, for both zero level and amplitude free in each case. This provides support for the hypothesis that a broad component is important; it may plausibly be attributed to inverse Compton or maybe to another component [11].

It is also possible to use the intensity images to construct spectra, taking the zero level to be the mean intensity at high latitude $\left(|b|>30^{\circ}\right)$. This gives the spectrum of the non-isotropic emission (Fig 3). The latitude range $|b|<5^{\circ}$ is chosen for this spectrum since this optimises the signal-to-noise ratio in this method. The result can be compared with that from model fitting, here illustrated by a free fit to gas/cosmic-ray interactions plus inverse Compton emission; for this method only points $>3 \mathrm{MeV}$ are shown since background temporal variations make it unreliable below this energy. Both maximum entropy and model fitting yield spectra which are harder than in [2]. The $|b|<20^{\circ}$ spectrum in $[2]$ is unreliable because of inadequate handling of the instrumental background. The present results are consistent with the higher 


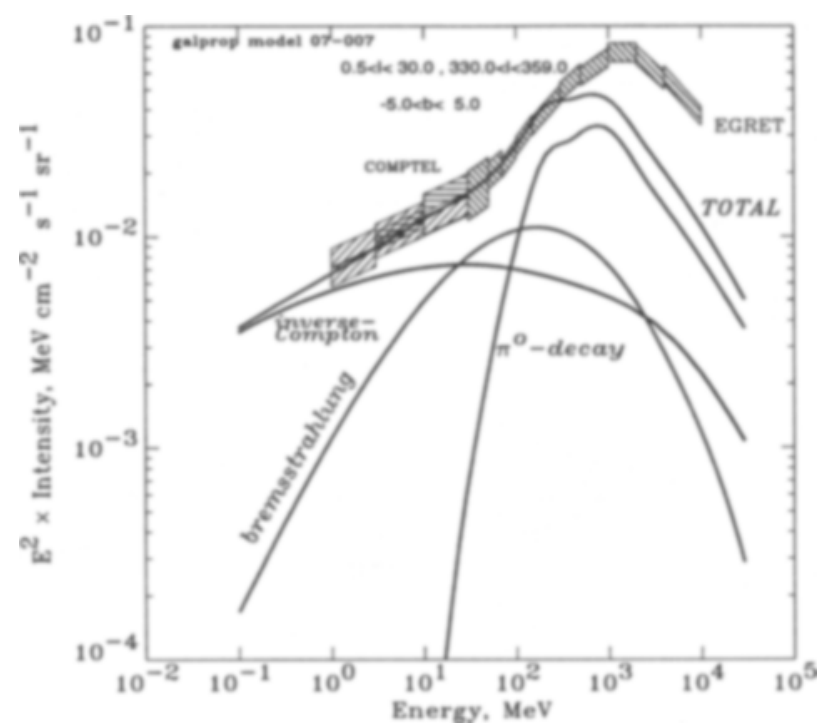

Fig. 3: The $\gamma$-ray spectrum for the inner Galaxy, $330^{\circ}<l<30^{\circ},|b|<5^{\circ}$.

///: from COMPTEL maximum entropy images using two different background scaling parameters $(0.90,0.95) ; \equiv$ : free fit of COMPTEL data to model based on gas + inverse Compton. $|1|$ : EGRET data [12]. Curves show the bremsstrahlung, inverse Compton and $\pi^{\circ}$-decay components and total for the physical model described in [10].

energy resolution spectra based on model fitting given in [11]. For comparison, a model from $[9,10]$ is shown, which fits the COMPTEL-EGRET spectrum from $1 \mathrm{MeV}$ to $300 \mathrm{MeV}$, beyond which the well known discrepancy with the canonical $\pi^{\circ}$-decay spectrum $[6]$ is evident.

\section{REFERENCES}

1. Strong A.W., Experimental Astronomy 6/4, 97 (1995)

2. Strong A.W. et al., $A \mathscr{E} A S$ 120, C381 (1996)

3. Strong A.W. et al., ESA SP-382, 533 (1997)

4. Diehl R, et al. Adv. Sp. Res 15, 5, 123 (1995)

5. Diehl R. et al. A\&A 298,445 (1995)

6. Hunter S.D. et al., ApJ 481, 205

7. Oberlack U. et al. (1997) these Proceedings

8. Strong A.W., A\& A 292, 82 (1994)

9. Strong A.W., Moskalenko I.V., Schönfelder V., 25th ICRC, OG8.1.1 (1997)

10. Strong A.W., Moskalenko I.V, in AIP Proc., (1997), these Proceedings

11. Bloemen H. et al., in AIP Proc., (1997), these Proceedings

12. Strong A.W., Mattox J.R., A $B A$ 308, L21 (1996) 